Originalien

Unfallchirurg 2022 $125: 305-312$

https://doi.org/10.1007/s00113-021-01015-5

Angenommen: 14. April 2021

Online publiziert: 8. Juni 2021

(c) Der/die Autor(en) 2021

\section{Redaktion}

W. Mutschler, München

H. Polzer, München

B. Ockert, München

\section{Zusatzmaterial online}

Die Online-Version dieses Beitrags (https://doi.org/10.1007/s00113-02101015-5) enthält zusätzliche Tabellen zur Kostenberechnung sowie detaillierte Informationen zur Methode, weitere Ergebnisse und weiterführende Literatur. Beitrag und Zusatzmaterial stehen Ihnen auf www.springermedizin.de zur Verfügung. Bitte geben Sie dort den Beitragstitel in die Suche ein, das Zusatzmaterial finden Sie beim Beitrag unter „Ergänzende Inhalte".

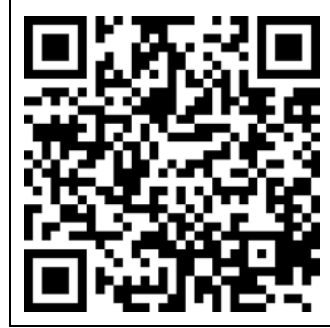

\section{Einleitung}

Eine Kernkompetenz eines überregionalen Traumazentrums (ÜTZ) ist die Versorgung von schwer verletzten und polytraumatisierten PatientInnen. Die Versorgung Schwerverletzter setzt eine interdisziplinäre Zusammenarbeit voraus und ist mit hohen Personal-, Material- und Vorhaltekosten verbunden. Notaufnahme, OP und Intensivstation eines ÜTZ müssen rund um die Uhr bereit sein, 2 Schwerverletzte zeitgleich bestmöglich $\mathrm{zu}$ versorgen, dafür sollte beispielsweise das Schockraumteam folgende Personen beinhalten: Basisteam

J. Fakler und G. Osterhoff haben zu gleichen Teilen zum Manuskript beigetragen.

Nikolas Schopow ${ }^{1}$ Anja Botzon ${ }^{2} \cdot$ Kristian Schneider $^{3}$ - Carolin Fuchs ${ }^{1} \cdot$ Christoph Josten ${ }^{1}$. Nikolaus von Dercks ${ }^{2}$. Johannes Fakler' · Georg Osterhoff'

'Klinik für Orthopädie, Unfallchirurgie und Plastische Chirurgie, Universitätsklinikum Leipzig, Leipzig, Deutschland

${ }^{2}$ Bereich 3 - Finanzen, Planung und Controlling, Universitätsklinikum Leipzig, Leipzig, Deutschland ${ }^{3}$ Klinik für Allgemein Orthopädie und Tumororthopädie, Universitätsklinikum Münster, Münster, Deutschland

\title{
Ist die Polytraumaversorgung im aG-DRG-System defizitär?
}

(sofort anwesend): $2^{\star}$ UnfallchirurgInnen, $1^{\star}$ AnästhesistIn, $3^{\star}$ Pflegekräfte, $1^{\star}$ Medizin-technische Radiologieassistenz (MTRA); erweitertes Team (zusätzlich innerhalb von $30 \mathrm{~min}$ ): OberärztInnen für spezielle Unfallchirurgie, Anästhesie, Neurochirurgie und Radiologie sowie $2^{\star}$ OP-Pflegekräfte; Spezialisten aus 12 weiteren Fachdisziplinen sollten vor Ort vorgehalten werden [24].

Diese Ressourcen werden für die Versorgung von Schwerverletzten bereitgehalten, dabei stehen Traumazentren vor der Herausforderung, Fälle unterschiedlicher Behandlungs- und Kostenintensität miteinander zu vereinbaren. Durch die Umstellung auf das German-Diagnosis-Related-Groups(G-DRG)-System in Deutschland im Jahr 2003 wurde ein Fallkostenpauschalsystem für die $\mathrm{Ab}$ rechnung der stationären PatientInnen eingeführt. Dies führte dazu, dass unterschiedliche Behandlungsfälle bei der Abrechnung mit den Krankenkassen in Gruppen gleicher Diagnosen zusammengefasst werden. Mit Einführung der aG-DRG im Jahr 2020 werden die Pflegepersonalkosten in den DRG-Fallpauschalen ausgegliedert und über ein krankenhausindividuelles Pflegebudget nach dem Selbstkostendeckungsprinzip finanziert.

In der klinischen Versorgung wurde ein Polytrauma ursprünglich definiert als das gleichzeitige Vorhandensein von Verletzungen verschiedener Körperregionen, von denen mindestens eine Verletzung oder aber die Kombination mehrerer Verletzungen als lebensbedrohlich einzuschätzen ist [1]. Wissenschaftlich existieren weitere Definitionen wie ein Injury Severity Score (ISS) $\geq 16$ oder die Berlin-Definition [2].

Im G-DRG-System wird die Diagnose Polytrauma zusammengefasst unter der „major diagnostic category“ (MDC) 21A und in 3 Basis-DRG unterteilt (Zusatzmaterial online: Tab. 1) [3-7].

Durch die Unterschiede in den Definitionen kommt es zu einer „Fehlgruppierung" von bis zu 30\% der polytraumatisierten Patienten in andere Hauptdiagnosen [8].

Bereits vor der Einführung der Fallkostenpauschalen wurden die ökonomischen Aspekte der Schwerverletztenversorgung national und international diskutiert $[9,10]$. Qvick et al. zeigten, dass die Umstellung von der tagessatzbasierten Abrechnung auf die fallkostenbasierte Abrechnung einer um $34 \%$ verringerten Vergütung der jeweiligen Versorgungsfälle gleichkommt [11].

In den bisher zu diesem Thema publizierten Studien wurden Verluste von -2565 bis $\mathrm{zu}-12.893 €$ je Fall aufgezeigt [12-17]. Dabei wurde zur Berechnung der tatsächlichen Behandlungskosten polytraumatisierter PatientInnen entweder eine Einzelkostenabrechnung entsprechend den Vorgaben des Instituts für das Entgeltsystem im Krankenhaus (InEK) durchgeführt, oder die Kosten wurden auf Behandlungszeiten in den einzelnen Zwischenschritten der Therapie heruntergerechnet. In keiner der bisherigen Veröffentlichungen wurden allerdings die Kosten der Organisation von Verlegungen externer PolytraumapatientInnen, die Zusatz- 


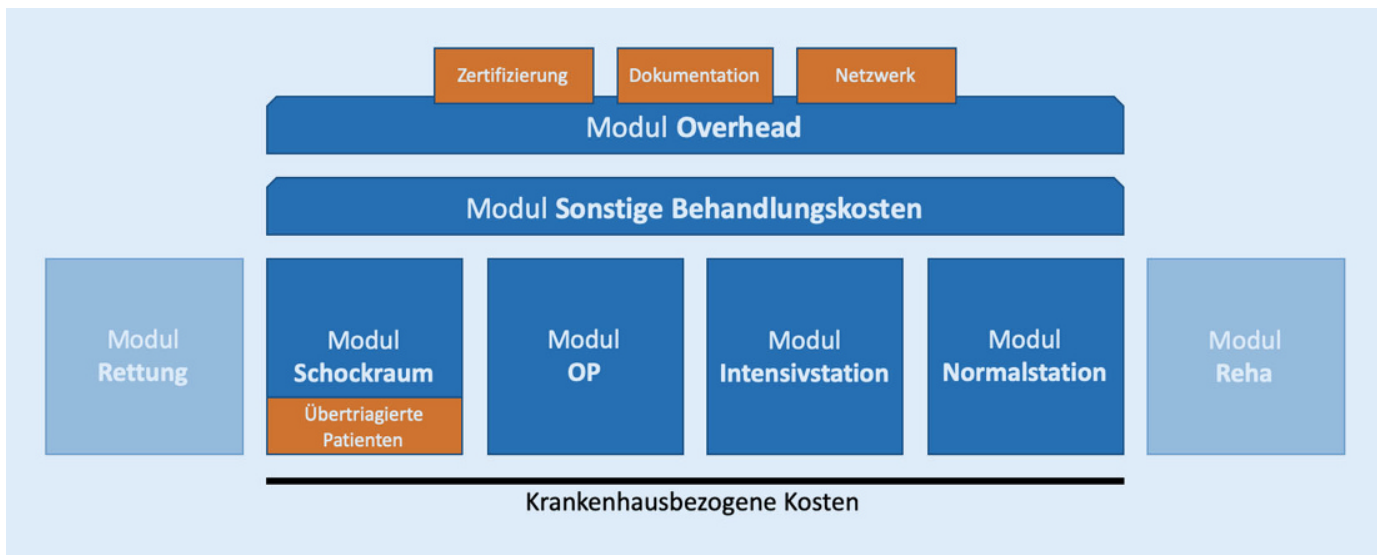

Abb. $1 \triangleleft$ Ergänztes Kostenmodel. (Modifiziert nach Pape et al. [18]) kosten durch „Übertriagierung“ und der Mehraufwand durch Dokumentationspflichten und Zertifizierungskosten hinreichend berücksichtigt.

Ziel dieser Arbeit ist daher eine Kosten-Erlös-Analyse der Behandlung Schwerverletzter, basierend auf einem nachvollziehbaren, detaillierteren und umfassenderen Modell. Für Krankenhäuser ohne Einzelkostenabrechnung ist eine modellbasierte Berechnung eine sinnvolle und umsetzbare Lösung zur Erfassung der Behandlungskosten. Vergleichend erfolgt die Berechnung entsprechend dem Kostenschätzer des TraumaRegister $\mathrm{DGU}^{\circledR}$ [12].

\section{Methode}

\section{Patientenkollektiv und Auswahlkriterien}

Eingeschlossen wurden alle PatientInnen, die im Jahr $2017 \mathrm{im}$ chirurgischen Schockraum der Zentralen Notaufnahme des Universitätsklinikums Leipzig (UKL) behandelt wurden. Eine detaillierte Kostenanalyse erfolgte an allen primär behandelten oder sekundär zuverlegten PatientInnen, die im TraumaRegister $\mathrm{DGU}^{\circledR}$ dokumentiert wurden. Im TraumaRegister $\mathrm{DGU}^{\circledR}$ wurden diejenigen Patienten dokumentiert, die nach Behandlung im Schockraum in einen OP oder auf die Intensivstation verlegt wurden und darüber hinaus mindestens einen ISS $\geq 9$ aufwiesen.

\section{Berechnung der Kosten}

Die Gesamtkosten der Schwerverletztenbehandlung setzen sich aus mehreren Kostenfaktoren zusammen. Für die hier vorliegende Berechnung wurde das Berechnungsmodell nach Pape et al. um die Kosten für übertriagierte Schockraumpatienten, Zertifizierungs-, Dokumentations- und Netzwerkkosten ergänzt (• Abb. 1; [18]). Alle dokumentierten Behandlungen, Aufenthaltszeiten, Materialien und Diagnosemittel wurden aus dem Krankenhausinformationssystem, der Auswertung des TraumaRegister DGU $^{\circledR}$ der PatientInnen des UKL sowie der klinikinternen Schockraumdokumentation erfasst und ausgewertet. Die detaillierte Berechnung der Module („Schockraum“, „OP“, „Intensivstation“, „Normalstation“, „Sonstige Behandlungskosten“ und „Overhead“ finden Sie im Zusatzmaterial online: ESM10: Detaillierte Methode, weitere Ergebnisse, weiterführende Literatur).

\section{Berechnung der Erlöse}

Die eingeschlossenen Fälle wurden in die DRG-Systeme 2017-2020 eingruppiert und die jeweiligen Relativgewichte berechnet. Die jeweiligen Zusatzentgelte wurden den Krankenhausfällen aufaddiert. Die Erlöse wurden anhand der Landesbasisfallwerte Sachsens (LBFW) von 2017-2020 berechnet (Zusatzmaterial online: Tab. 6). Die Diagnose DRG B61B (bestimmte akute Erkrankungen und Verletzungen des Rückenmarks ohne komplexen Eingriff oder mehr als 13 Belegungstage oder nicht wegverlegt) ist nicht in dem Fallpauschalenkatalog vergüteter, vollstationärer Leistungen enthalten. Es handelt sich um ein krankenhausindividuelles Entgelt nach $\$ 6$ Abs. 1 Satz 1 des Krankenhausentgeltgesetzes. Für diese Diagnose wurde der am Universitätsklinikum Leipzig vereinbarte Betrag als Rechnungsgrundlage in Höhe von 12.157,93€ angenommen.

Die fiktiven Erlöse für die Jahre 2018-2020 wurden mithilfe von Übergangsgroupern berechnet (Fa. ID Berlin, Berlin, Deutschland).

Die Berechnungsmethode und Kalkulationsdaten des Kostenschätzers im TraumaRegister $\mathrm{DGU}^{\circledR}$ finden Sie im Zusatzmaterial online: ESM10: Detaillierte Methode, weitere Ergebnisse, weiterführende Literatur [12].

\section{Statistische Analysen}

Kontinuierliche Daten wurden als Mittelwert mit Standardabweichung dargestellt, diskrete Daten als absolute und prozentuale Zahl. Die Berechnung der Mittelwerte, Standardabweichung und Signifikanztestung ( $t$-Test und Chi-QuadratTest) erfolgten in SPSS (Fa. IBM, Armonk, NY, USA). Als statistisch signifikant wurden $p$-Werte $<0,05$ gewertet.

\section{Ergebnisse}

\section{Patientenkollektiv}

Im Beobachtungszeitraum wurden 834 PatientInnen primär oder sekundär über den Schockraum aufgenommen (• Abb. 2). Davon wurden 258 PatientInnen als schwer verletzt eingestuft, im TraumaRegister DGU ${ }^{\circledR}$ (ISS 9-15+ ITS 
oder ISS $\geq 16$ ) entsprechend dokumentiert und in der vorliegenden Arbeit in der Gruppe „Gesamt“ zusammengefasst. Diese PatientInnen wurden für die Analyse in 2 Kohorten weiter aufgeteilt: nichtpolytraumatisierte Schwerverletzte „ISS 9-15+ ITS“ ( $n$ 72; $\varnothing$ ISS 11,9 $\pm 1,8$; ISS $_{\min } 9-$ ISS $\left._{\max } 15\right)$ und polytraumatisierte Patienten „ISS $\geq 16$ “ ( $n$ 186; $\varnothing$ ISS $27,7 \pm 13,5$; ISS $_{\min } 16-$ ISS $_{\max }$ 75). Zwischen diesen beiden Gruppen gab es keinen signifikanten Unterschied bei Alter ( $\left.p_{\text {ISS }} 0,188\right)$, Geschlecht ( $\left.p_{\text {ISS }} 0,539\right)$ und Vorerkrankungen (ASA-Score) ( $p_{\text {ISS }}$ 0,136). Zusätzlich wurden zur DRGbezogenen Betrachtung alle PatientInnen mit der Hauptdiagnose MCD 21A gruppiert in der Gruppe „DRG-Polytrauma" ( $n$ 59; $\varnothing$ ISS 32,4 $\pm 16,4$; ISS $_{\text {min }}$ 13-ISS max $_{\max }$ 75). Im Vergleich zwischen der Gruppe „DRG-Polytrauma“ und den restlichen PatientInnen aus der Gruppe "Gesamt" besteht ein signifikanter Unterschied im Altersdurchschnitt ( $p_{\text {DRG }}$ 0,035). Kein signifikanter Unterschied besteht bei der Geschlechterverteilung ( $\left.p_{\text {DRG }} 0,749\right)$ und zwischen den ASAScores $\left(p_{\text {DRG }} 0,103\right)$. In der Gruppe „kein Polytrauma" sind die PatientInnen enthalten, die zwar über den chirurgischen Schockraum, aber ohne entsprechendes Verletzungsmuster angenommen wurden ( $n$ 553; $\varnothing$ ISS $4,0 \pm 4,3$; ISS $_{\min }$ $0-$ ISS $\left._{\max } 15\right)$. Wenn nicht anders angegeben, beziehen sich die im weiteren Fließtext angegebenen Werte auf das Jahr 2020. Dargestellt werden jeweils die Gesamtkosten/-erlöse (Kosten/Erlöse pro PatientIn) der Gruppe "Gesamt“ (- Abb. 3), die einzelnen Kostenpunkte und Subgruppen des Patientenkollektivs sind im Zusatzmaterial online: Tab. 7 aufgeschlüsselt.

\section{Modul "Schockraum"}

Trotz der variierenden Behandlungsintensität bestand klinisch und statistisch kein signifikanter Unterschied in der durchschnittlichen Dauer der Schockraumbehandlung zwischen den Gruppen (35-40 min, $p_{\text {ISS }} 0,411$ und $p_{\text {DRG }}$ 0,365). Aus der Gruppe „ISS 9-15+ ITS“ war kein/keine PatientIn bei der Übergabe im Schockraum reanimationspflichtig, und nur in $6 \%$ der Fälle wurde eine Thoraxsaugdrainage

Unfallchirurg 2022 $\cdot 125: 305-312$ https://doi.org/10.1007/s00113-021-01015-5

(c) Der/die Autor(en) 2021

N. Schopow · A. Botzon · K. Schneider · C. Fuchs · C. Josten · N. von Dercks · J. Fakler · G. Osterhoff

Ist die Polytraumaversorgung im aG-DRG-System defizitär?

\section{Zusammenfassung}

Hintergrund. Die interdisziplinäre Versorgung von polytraumatisierten PatientInnen ist personal- und ressourcenaufwendig. Seit der Einführung des G-DRG-Systems 2003 in Deutschland wurde in den meisten Untersuchungen ein finanzielles Defizit in der Schwerstverletztenversorgung festgestellt. Ziel dieser Studie war es, Auswirkungen des 2020 neu eingeführten aG-DRG-Systems auf die Kostendeckung in der Schwerverletztenbehandlung zu analysieren. Erstmals wurden auch die Kosten für Organisation, Zertifizierung und Dokumentation sowie die Kosten für nicht schwer verletzte SchockraumpatientInnen betrachtet.

Methodik. Eingeschlossen wurden alle PatientInnen, die im Jahr 2017 im chirurgischen Schockraum der Zentralen Notaufnahme des Universitätsklinikums Leipzig behandelt wurden. Für die Analyse wurden das Kostenmodell nach Pape et al. um die Module Organisation, Dokumentation und Zertifizierung ergänzt sowie die Kosten für "übertriagierte" PatientInnen betrachtet. Es erfolgte die Berechnung der Kosten in den Jahren 2017-2020 im Vergleich der jeweiligen Erlöse.

Ergebnisse. Es wurden 834 PatientInnen im Schockraum behandelt. Die 258 schwer verletzten PatientInnen wurden in 3 Gruppen

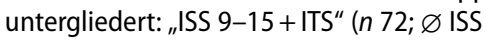
11,9 ; Kosten/Patientln $14.715 €$ ), "ISS $\geq 16^{\prime \prime}$ ( $n$ 186; $\varnothing$ ISS 27,7; Kosten/PatientIn 30.718€) und "DRG-Polytrauma“ ( $n$ 59; $\varnothing$ ISS 32,4; Kosten/PatientIn $26.102 €$ ). Schlussfolgerung. Die Polytraumaversorgung im aG-DRG 2020 ist defizitär. Insgesamt entstand im Jahr 2020 ein Defizit von $5858 €$ pro schwer verletztem/verletzter Patientln.

Schlüsselwörter

Schockraum · Schwerstverletzt · Ökonomische Evaluation - Finanzielle Analyse . Behandlungskosten

\section{Is polytrauma treatment in deficit in the aG-DRG system?}

\section{Abstract}

Background. The interdisciplinary care of severely injured patients is staff and resource intensive. Since the introduction of the G-DRG system in Germany in 2003, most studies have identified a financial deficit in the care of severely injured patients. The aim of this study was to analyze the effects of the new aG-DRG system introduced in 2020 on cost recovery in the treatment of severely injured patients. For the first time, the costs for organization, certification and documentation as well as the costs for non-seriously injured shock room patients were included.

Methods. All patients who were treated in the surgical shock room of the emergency department of the Leipzig University Hospital in 2017 were included. For the analysis, the cost model according to Pape et al. was extended by the module organization, documentation and certification and for the first time the costs for overtriaged patients were considered. A cost calculation was performed for the years 2017-2020 as well a comparison with the respective earnings. Results. A total of 834 patients were treated in the shock room and 258 severely injured patients were divided into 3 groups: ISS $9-15+$ ICU $(n$ 72; $\varnothing$ ISS 11.9; costs per patient $14,715 €$ ),ISS $\geq 16$ ( $n$ 186; $\varnothing$ ISS 27.7; costs per patient $30,718 €$ ) and DRG polytrauma ( $n$ 59; $\varnothing$ ISS 32.4; costs per patient 26,102 $€$ ).

Conclusion. Polytrauma care under the aGDRG 2020 is in deficit. Overall, in 2020 a deficit of $5858 €$ per severely injured patient resulted.

\section{Keywords}

Shock room - Severely injured - Economic evaluation - Financial analysis - Treatment costs

angelegt. Demgegenüber waren $17 \%$ der PatientInnen der Gruppe „DRGPolytrauma" reanimationspflichtig, und $27 \%$ benötigten mindestens eine Thoraxsaugdrainage. In der Schockraumphase erhielten keiner/keine der PatientInnen der Gruppe „ISS 9-15+ ITS“, aber 13\% der Gruppe „ISS $\geq 16$ “ und $29 \%$ der Gruppe „DRG-Polytrauma“ eine Bluttransfusion.

Für die Behandlung im Schockraum entstanden Kosten von 412.101€ (1597€/ 


\begin{tabular}{|c|c|c|c|c|c|c|}
\hline Kohorte & & Gesamt & ISS 9-15 + ITS & ISS > 16 & DRG-Polytrauma & Kein Polytrauma \\
\hline Anzahl der Patienten & $n$ & 258 & 72 & 186 & 59 & 553 \\
\hline Geschlecht männlich & $\%(n)$ & $71,71 \%(185)$ & $75,00 \%(54)$ & $70,43 \%(131)$ & $72,88 \%(43)$ & $64,01 \%(354)$ \\
\hline Alter (Jahre) & $\mathrm{MW}(\mathrm{SD})$ & $53,63(23,34)$ & $50,56(23,63)$ & $54,82(23,19)$ & $47,56(22,07)$ & $43,83(22,69)$ \\
\hline ASA & $\mathrm{MW}(\mathrm{SD})$ & $1,83(0,83)$ & $1,67(0,80)$ & $1,89(0,84)$ & $1,59(0,70)$ & \\
\hline Primäre Versorgung & $\%(n)$ & $65,12 \%(168)$ & $63,89 \%(46)$ & $65,59 \%(122)$ & $72,88 \%(43)$ & \\
\hline Stumpfes Trauma & $\%(n)$ & $95,74 \%(247)$ & $94,44 \%(68)$ & $96,24 \%$ (179) & $98,31 \%(58)$ & \\
\hline ISS & $\mathrm{MW}(\mathrm{SD})$ & $23,27(13,49)$ & $11,86(1,86)$ & $27,69(13,47)$ & $32,41(16,42)$ & $4,00(4,29)$ \\
\hline AIS Kopf & $\mathrm{MW}(\mathrm{SD})$ & $2,30(1,82)$ & $1,38(1,32)$ & $2,67(1,86)$ & $2,00(1,74)$ & \\
\hline AIS Kopf ( $\geq 3$ ) & $\%(n)$ & $51,16 \%(132)$ & $31,94 \%(23)$ & $58,6 \%(109)$ & $40,68 \%(24)$ & \\
\hline AIS Gesicht & $\mathrm{MW}(\mathrm{SD})$ & $0,53(0,95)$ & $0,49(0,82)$ & $0,54(1,00)$ & $0,51(1,01)$ & \\
\hline AIS Gesicht ( $\geq 3$ ) & $\%(n)$ & $6,20 \%(16)$ & $2,78 \%(2)$ & $7,53 \%(14)$ & $8,47 \%(5)$ & \\
\hline AIS Thorax & $\mathrm{MW}(\mathrm{SD})$ & $1,78(1,80)$ & $1,00(1,36)$ & $2,08(1,87)$ & $2,92(1,69)$ & \\
\hline AIS Thorax ( $\geq 3$ ) & $\%(n)$ & $43,41 \%(112)$ & $27,78 \%(20)$ & $49,46 \%(92)$ & $64,41 \%(38)$ & \\
\hline AIS Abdomen & $\mathrm{MW}(\mathrm{SD})$ & $0,80(1,39)$ & $0,33(0,86)$ & $0,98(1,51)$ & $1,41(1,63)$ & \\
\hline AIS Abdomen ( $\geq 3$ ) & $\%(n)$ & $15,12 \%(39)$ & $5,56 \%(4)$ & $18,82 \%(35)$ & $28,81 \%(17)$ & \\
\hline AIS Extremitäten & $\mathrm{MW}(\mathrm{SD})$ & $1,56(1,59)$ & $1,31(1,26)$ & $1,66(1,70)$ & $2,85(1,47)$ & \\
\hline AIS Extremitäten ( $\geq 3$ ) & $\%(n)$ & $31,78 \%(82)$ & $23,61 \%(17)$ & $34,95 \%(65)$ & $67,80 \%(40)$ & \\
\hline AIS Weichteile & $\mathrm{MW}(\mathrm{SD})$ & $0,47(0,68)$ & $0,49(0,56)$ & $0,47(0,72)$ & $0,49(0,80)$ & \\
\hline AIS Weichteile ( $\geq 3$ ) & $\%(n)$ & $1,16 \%(3)$ & $0 \%(0)$ & $1,61 \%(3)$ & $1,69 \%(1)$ & \\
\hline GCS im SR & $\mathrm{MW}(\mathrm{SD})$ & $11,07(5,27)$ & $13,71(2,97)$ & $10,05(5,60)$ & $9,20(5,72)$ & \\
\hline RRsys im SR (mmHg) & $\mathrm{MW}(\mathrm{SD})$ & $133,32(37,05)$ & $141,36(29,01)$ & $130,22(39,36)$ & $114,84(46,02)$ & \\
\hline Puls im SR $\left(\mathrm{min}^{-1}\right)$ & $\mathrm{MW}(\mathrm{SD})$ & $89,73(25,26)$ & $89,66(18,98)$ & $89,76(27,38)$ & $85,24(31,80)$ & \\
\hline Sauerstoffsättigung im SR [\%] & $\mathrm{MW}(\mathrm{SD})$ & $96,34(11,58)$ & $97,54(2,66)$ & $95,87(13,56)$ & $93,30(19,75)$ & \\
\hline Intubiert bei Ankunft im SR & $\%(n)$ & $27,91 \%(72)$ & $6,94 \%(5)$ & $36,02 \%(67)$ & $44,07 \%(26)$ & \\
\hline Hb-Wert im SR (mmol/l) & $\mathrm{MW}(\mathrm{SD})$ & $7,92(1,36)$ & $8,46(0,96)$ & $7,71(1,43)$ & $7,17(1,66)$ & \\
\hline BE im SR (mmol/l) & $\mathrm{MW}(\mathrm{SD})$ & $-1,21(5,39)$ & $0,53(3,51)$ & $-1,89(5,83)$ & $-3,35(7,71)$ & \\
\hline Patienten mit EK-Transfusion im SR & $\%(n)$ & $9,30 \%(24)$ & $0 \%(0)$ & $12,90 \%(24)$ & $28,81 \%(17)$ & \\
\hline Reanimation im SR & $\%(n)$ & $5,43 \%(14)$ & $0 \%(0)$ & $7,53 \%(14)$ & $16,95 \%(10)$ & \\
\hline Anlage TSD im SR & $\%(n)$ & $9,69 \%(25)$ & $5,56 \%(4)$ & $11,29 \%(21)$ & $27,12 \%(16)$ & \\
\hline Hämostase-Therapie im SR & $\%(n)$ & $11,63 \%(30)$ & $2,78 \%(2)$ & $15,05 \%(28)$ & $30,51 \%(18)$ & \\
\hline Dauer des SR (min) & $\mathrm{MW}(\mathrm{SD})$ & $37,33(24,34)$ & $35,33(19,76)$ & $38,10(25,90)$ & $40,14(27,62)$ & \\
\hline Behandlungstage auf ITS (Tage) & $\mathrm{MW}(\mathrm{SD})$ & $7,88(12,83)$ & $2,72(4,78)$ & $9,87(14,34)$ & $7,09(8,85)$ & \\
\hline Beatmungstage auf ITS (Tage) & $\mathrm{MW}(\mathrm{SD})$ & $3,03(8,04)$ & $0,73(4,09)$ & $3,91(8,96)$ & $2,14(4,37)$ & \\
\hline Behandlungstage insg. (Tage) & $\mathrm{MW}(\mathrm{SD})$ & $18,60(17,99)$ & $11,25(7,75)$ & $21,10(19,73)$ & $18,91(19.01)$ & \\
\hline Outcome "gut erholt" & $\%(n)$ & $32,95 \%(85)$ & $52,78 \%(38)$ & $25,27 \%(47)$ & $18,64 \%(11)$ & \\
\hline Outcome "mässig behindert" & $\%(n)$ & $35,27 \%(91)$ & $40,28 \&(29)$ & $33,33 \%(62)$ & $44,07 \%(26)$ & \\
\hline Outcome "schwer behindert" & $\%(n)$ & $16,67 \%(43)$ & $4,17 \%(3)$ & $21,51 \%(40)$ & $11,86 \%(7)$ & \\
\hline Outcome "tot" & $\%(n)$ & $14,34 \%(37)$ & $2,278 \%(2)$ & $18,82 \%(35)$ & $25,42 \%(15)$ & \\
\hline
\end{tabular}

Abb. 2 ॥ Gruppencharakteristika. ASA ASA-Klassifikation, ISS Injury Severity Score, AIS Abbreviated Injury Scale, GCS Glasgow Coma Scale, $B E$ "base excess", SR Schockraum, TSD Thoraxsaugdrainage, ITS Intensivstation

PatientIn) (• Abb. 3). Dabei enthalten sind $220.284 €(845 €)$ für den überschüssigen Personaleinsatz bei „übertriagierten" PatientInnen. Dies entspricht einem Anteil von 53,4\% der gesamten Modulkosten bzw. 3,3\% der Gesamtkosten.

\section{Modul "OP“}

Ein signifikanter Unterschied innerhalb der Subgruppen zeigte sich bei der Anzahl der durchgeführten Operationen. PatientInnen der Gruppe „ISS 9-15+ITS“ wurden im Durchschnitt 1,2-mal operiert, gegenüber 2,0 Operationen in der Gruppe "ISS $\geq 16$ “ ( $p_{\text {ISS }}$ 0,038 ) und 2,6 Operationen in der Gruppe „DRG-Polytrauma“ ( $\left.p_{\text {DRG }} 0,004\right)$. Die Dauer pro Operation lag bei $81-86 \mathrm{~min}$ pro Eingriff in den Subgruppen. Insge- 


\begin{tabular}{|c|c|c|c|c|}
\hline & \multicolumn{2}{|l|}{ Kostenpunkte } & $\begin{array}{l}\text { Gruppe } \\
\text { "Gesamt" }\end{array}$ & $\begin{array}{l}\text { pro Patient } \\
\text { "Gesamt" }\end{array}$ \\
\hline $\begin{array}{l}\text { Anzahl der } \\
\text { Patienten }\end{array}$ & & $n$ & 258 & \\
\hline $\begin{array}{l}\text { Relativgewicht } \\
2020^{a}\end{array}$ & & & 1116,1 & 4,5 \\
\hline \multirow{7}{*}{ Schockraum } & Dauer & $\min$ & 9631 & 37,3 \\
\hline & Personal ÄD & $€$ & $111.818 €$ & $433 €$ \\
\hline & Personal PD & $€$ & $16.111 €$ & $62 €$ \\
\hline & Umverteilung Personalkosten & $€$ & $220.284 €$ & $854 €$ \\
\hline & Infrastruktur & $€$ & $34.623 €$ & $134 €$ \\
\hline & Verbrauchsgüter & $€$ & $29.265 €$ & $113 €$ \\
\hline & Modulkosten & $€$ & $412.101 €$ & $1.597 €$ \\
\hline \multirow{8}{*}{$\mathrm{OP}$} & Anzahl der OPs & $\mathrm{n}$ & 457 & 1,8 \\
\hline & Schnitt-Naht-Zeit & $\min$ & 37531 & 145,5 \\
\hline & Anästhesie & $€$ & $355.232 €$ & $1.377 €$ \\
\hline & Peronsal ÄD Chirurgie & $€$ & $146.570 €$ & $568 €$ \\
\hline & Peronsal PD Chirurgie & $€$ & $67.493 €$ & $262 €$ \\
\hline & Material und Implantate & $€$ & $496.356 €$ & $1.924 €$ \\
\hline & Infrastruktur & $€$ & $7.893 €$ & $31 €$ \\
\hline & Modulkosten & $€$ & $1.073 .544 €$ & $4.161 €$ \\
\hline \multirow{3}{*}{ Intensivstation } & $\begin{array}{l}\text { Behandlungstage } \\
\text { Intensivstation }\end{array}$ & $d$ & 2032 & 7,9 \\
\hline & Beatmungstage & $d$ & 770 & 3,0 \\
\hline & Modulkosten & $€$ & $3.011 .891 €$ & $11.674 €$ \\
\hline \multirow{2}{*}{ Normalstation } & Behandlungstage & $d$ & 2654 & 10,3 \\
\hline & Modulkosten & $€$ & $1.363 .949 €$ & $5.287 €$ \\
\hline \multirow{7}{*}{$\begin{array}{l}\text { Sonstige } \\
\text { Behandlung }\end{array}$} & Radiologie und Nuklearmedizin & $€$ & $331.156 €$ & $1.284 €$ \\
\hline & $\mathrm{C} / \mathrm{M} / \mathrm{V} / \mathrm{P} / \mathrm{I}$ & $€$ & $136.190 €$ & $528 €$ \\
\hline & Transfusionspräparate & $\mathrm{n}$ & 1034 & 4,0 \\
\hline & Transfusionsmedizin & $€$ & $165.641 €$ & $642 €$ \\
\hline & Ärztliche Konsile & $€$ & $46.133 €$ & $179 €$ \\
\hline & $\mathrm{P} / \mathrm{E} / \mathrm{L}$ & $€$ & $76.835 €$ & $298 €$ \\
\hline & Modulkosten & $€$ & $755.956 €$ & $2.930 €$ \\
\hline \multirow{3}{*}{ Overhead } & Z/D/O & $€$ & $125.223 €$ & $485 €$ \\
\hline & Kosten Traumahandy & $€$ & $30.349 €$ & $118 €$ \\
\hline & Modulkosten & $€$ & $155.572 €$ & $603 €$ \\
\hline \multirow{7}{*}{ Gesamt } & Gesamtkosten & $\underline{\epsilon}$ & 6.773.014€ & $\underline{26.252 €}$ \\
\hline & \begin{tabular}{|l|} 
Kostenschätzer \\
\end{tabular} & $€$ & $6.811 .194 €$ & $26.400 €$ \\
\hline & Gesamterlös & $\underline{€}$ & $\underline{5.261 .526 €}$ & $\underline{20.394 €}$ \\
\hline & $\overline{\text { Teilerlös ohne Pflege und ZE/ZP }}$ & $\bar{\epsilon}$ & $3.729 .592 €$ & $\overline{14.456 €}$ \\
\hline & \begin{tabular}{|l|} 
Teilerlös Pflege \\
\end{tabular} & $€$ & $1.289 .213 €$ & $4.997 €$ \\
\hline & Teilerlös Zusatzentgelte & $€$ & $121.143 €$ & $470 €$ \\
\hline & Gesamtergebnis & $€$ & $\underline{-1.511 .488 €}$ & $-5.858 €$ \\
\hline
\end{tabular}

Abb. 3 A Kosten und Erlöse im Jahr 2020. Ausführliche Darstellung im Zusatzmaterial online: Tab. 7. ${ }^{a}$ Die 10 Patienten mit der Diagnose B61B sind nicht berücksichtigt. Z/D/O-Kosten 4,89\%.C/M/V/P/l klinische Chemie, Mikrobiologie, Virologie, Pathologie und Immunologie, $P / E / L$ Physio-/Ergotherapie und Logopädie, Z/D/O Zertifizierung-, Dokumentations- und Organisationskosten

samt entstanden Operationskosten von $1073.544 €(4161 € /$ PatientIn).

\section{Modul "Intensivstation"}

Zwischen den Gruppen „ISS9-15+ ITS“ und „ISS $\geq 16$ “ bestehen signifikante Unterschiede in der Behandlungsdauer $\left(2,72-9,87\right.$ Tage, $p_{\text {ISS }}<0,001, p_{\text {DRG }}$ $0,672)$ und der invasiven Beatmungsdauer $\left(0,71-3,97\right.$ Tage, $p_{\text {ISS }} 0,004$, p $\left._{\text {DRG }} 0,345\right)$ den Gruppen ( $p_{\text {ISS }} 0,311$, $p_{\text {DRG }} 0,456$ ). Es entstanden Kosten in Höhe von 1.363.949€ (5.287 €/PatientIn).

\section{Modul "Sonstige Behandlungskos- ten"}

Für radiologische und nuklearmedizinische Untersuchungen entstanden Kosten in Höhe von $331.157 €(1284 € /$ PatientIn). Für Laboruntersuchungen und Bereitstellung von Transfusionspräparaten $301.832 €(1170 €)$, für ärztliche Konsile $46.133 €(179 €)$ und für die Behandlung durch Ergo-/PhysiotherapeutInnen sowie LogopädInnen entstanden Kosten in Höhe von $76.835 €(298 €)$ (anteilige Verteilung: Abb. im Zusatzmaterial online:ESM10: Detaillierte Methode, weitere Ergebnisse, weiterführende Literatur).

\section{Modul "Overhead"}

Die Arbeitsbelastung des Trauma-Leaders für die Annahme von Schwerverletzten und Organisation externer Zuverlegung betrug $54 \mathrm{~min} /$ Tag. Daraus resultieren Arbeitskosten von $30.349 €$ (118€/ PatientIn). Des Weiteren ergeben sich Kosten durch Dokumentation und Zertifizierung von $125.223 €(485 €)$. Insgesamt entstanden Kosten von $155.572 €$ (603€).

\section{Gesamtkosten}

Die Kosten des Moduls „Schockraum“ (1556-1656€/PatientIn) unterschieden sich in den Subgruppen nicht wesentlich. Die Kosten der Module „OP“ (2609-6018€), „Intensivstation“ (3705-14.759€), „Normalstation“ (4681-5903€) und „Sonstige Behandlungskosten“ (1561-3460€) unterscheiden sich deutlich. Die Kosten im Modul „Overhead“ lagen bei $603 € /$ PatientIn. Die Gesamtkosten summierten sich auf $6.773 .014 €(26.252 €)$. Die Kosten der Subgruppen betragen: „ISS 9-15+ ITS“ $14.715 € /$ PatientIn, „ISS $\geq 16$ “ $30.718 €$ und „DRG-Polytrauma“ 26.102€. Mit $44,5 \%$ macht die intensivmedizinische Behandlung den größten Anteil der Gesamtkosten aus (•Abb. 4).

\section{Kostenschätzer im TraumaRegister $\mathrm{DGU}^{\circledR}$}

Entsprechend der Multiplikatormethode entstanden im Durchschnitt Kosten 


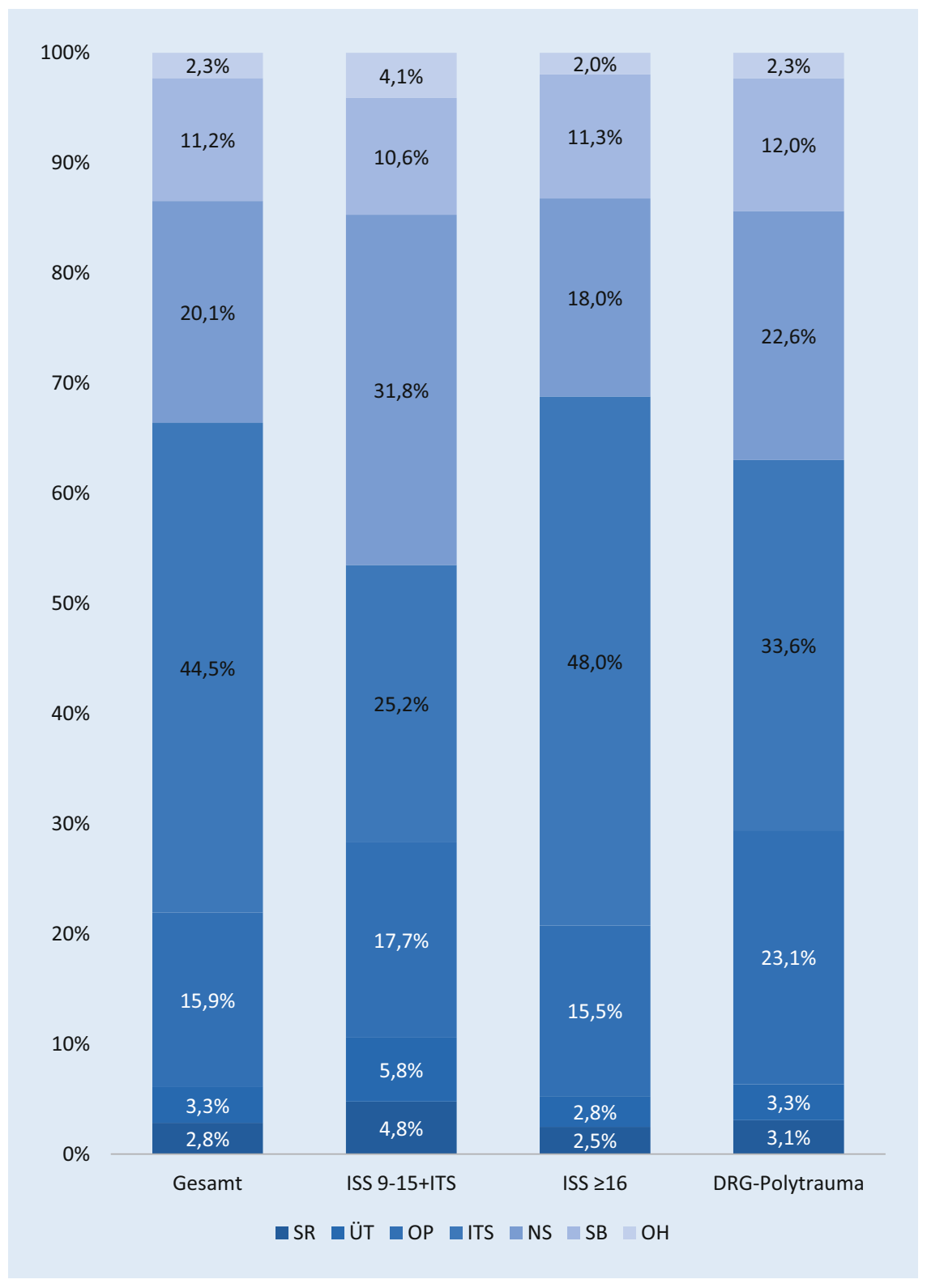

Abb. 4 A Anteilige Modulkostenverteilung. Die Kosten der Schockraumbehandlung (SR) wurden getrennt von den Kosten durch Übertriage (ÜT) dargestellt. OP Modul OP, ITS Modul „Intensivstation“, NS Modul "Normalstation", SB Modul "Sonstige Behandlungskosten", OH Modul „Overhead"

bei der Versorgung der schwer verletzten PatientInnen in Höhe von 6.811.194€ (26.400€/PatientIn).

\section{Gesamterlöse und Vergleich}

Die simulierten Erlöse des UKL lagen bei $5.261 .526 €(20.394 € /$ PatientIn). In den Subgruppen: „ISS 9-15+ITS“ $23.902 €$ /PatientIn, „ISS $\geq 16$ “ $19.035 €$ und „DRG-Polytrauma“ $24.120 €$. Ein Anteil von $24,5 \%$ der Erlöse entfällt auf die 2020 ausgegliederten Pflegeperso- nalkosten und 2,3\% auf Zusatzentgelte (•Abb.5).

Im Vergleich besteht eine deutliche Differenz zwischen den Gesamtkosten und den Gesamterlösen. Das Defizit beträgt $1.511 .488 €(5858 €)$.

\section{Diskussion}

Ziel dieser Studie war eine aktuelle Kosten-Erlös-Analyse der Behandlung schwer verletzter PatientInnen, basierend auf einem erweiterten Model nach
Pape et al., welches auch Kosten der Organisation von Verlegungen, Zusatzkosten durch „Übertriagierung" und den Mehraufwand durch Dokumentationspflichten und Zertifizierungskosten berücksichtigt sowie die Erlösstruktur des neu eingeführten aG-DRG-Systems [18].

Am UKL wird für das Jahr 2020 ein Defizit von 1.511.488€ durch die Versorgung von schwer verletzter PatientInnen kalkuliert. Allerdings bestehen deutliche Unterschiede in den Subgruppen; es entstehen Gewinne von $9187 € /$ PatientIn der Gruppe „ISS 9-15+ITS“ und Defizite von $11.683 €$ bzw. $1981 €$ in den Gruppen „ISS $\geq 16$ “ bzw. „DRG-Polytrauma“. Eine Subgruppenanalyse der PolytraumaDRG finden Sie im Zusatzmaterial online: Tab. 9.

Ab dem Jahr 2020 wird der Fallpauschalenkatalog um den Pflegeerlöskatalog erweitert und die Bezeichnung auf „aG-DRG-Katalog“ geändert. Die Höhe des Pflegeentgeltes pro Tag berechnet sich auf Basis eines krankenhausindividuellen Pflegeentgeltwertes. Diese Änderung ist in den Erlösen 2020 bereits berücksichtigt. Ein Erlösanteil von fast $25 \%$ entfällt dabei auf die ausgegliederten Pflegepersonalkosten.

Die Ergebnisse zeigen, dass die Versorgung von Schwerverletzten 17 Jahre nach Einführung des G-DRG-Systems weiterhin deutlich defizitär erfolgt. Damit bestätigt sich der positive Trend in der Vergütung von Polytraumata der vorhergegangenen Untersuchungen nicht [12-17]. Die jährliche Kostensteigerung 2017-2020 beträgt 4,4\%, hingegen wachsen die Erlöse nur um $1,1 \%$ pro Jahr (vgl. 2,9\% Kostensteigerung in deutschen Krankenhäusern pro Behandlungsfall (Zusatzmaterial online: Tab. 2)) [19-22]. Hierdurch droht sich das Defizit weiter zu vergrößern.

Alleine durch den Personalaufwand bei der Versorgung übertriagierter PatientInnen sind im Modul Schockraum Kosten in Höhe von $220.284 €$ entstanden, was einem Anteil von 3,3\% der Gesamtbehandlungskosten entspricht. Dies liegt an den weitgefassten Kriterien zur Aktivierung des Schockraumalarms [23]. Hierdurch wird zwar eine große Sensitivität, aber keine gute Spezifität 


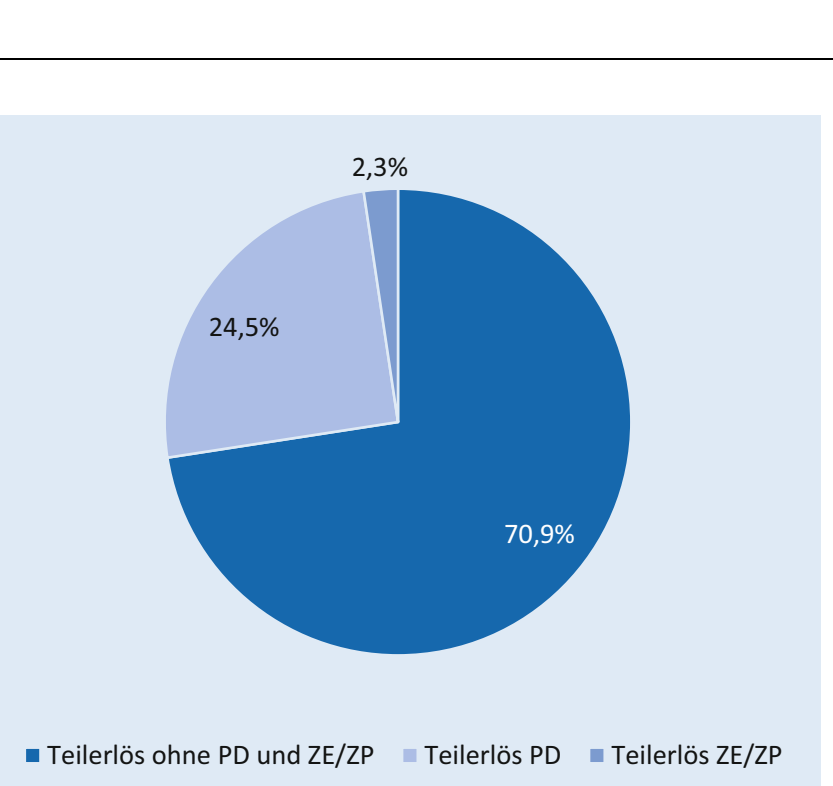

Abb. $5<$ Anteil der Teilerlöse am Gesamterlös. PD Pflegepersonalkosten, ZE/ZP Zusatzentgelte

erreicht, sodass $69 \%$ aller über den Schockraum aufgenommenen Patienten kein Polytrauma im definierenden Sinne darstellen. Ausstehend bleibt dabei die Möglichkeit zur Personalkostenreduktion durch eine gestaffelte Schockraumaktivierung, wie in der 3. erweiterten Auflage des Weißbuch der DGU für überregionale Traumazentren gefordert und am UKL zwischenzeitlich umgesetzt („gelber Alarm“ für wache und kreislaufstabile PatientInnen mit einem reduzierten Kernteam und „roter Alarm“ für bewusstlose oder kreislaufinstabile PatientInnen mit allen Teammitgliedern) [24].

Aktuell sind in den Fallpauschalen des DRG-Systems die Versorgung der übertriagierten PatientInnen und die Kosten für Dokumentation, Zertifizierung und Organisation nicht hinreichend abgebildet. Auch wenn man den Versorgungsaufwand ohne diese Kosten betrachtet, ergibt sich ein Defizit von 1.135.632€ (4402€/PatientIn).

Die Möglichkeiten zur Reduktion laufender Krankenhauskosten in der Versorgung von schwer verletzten PatientInnen sind begrenzt. Taheri et al. zeigten, dass Einsparungen im Bereich der intensivmedizinischen Versorgung von schwer verletzten PatientInnen im Bereich der variablen und damit direkt beeinflussbaren Kosten die geringste Summe der Ausgaben darstellen [25, 26]. Jedoch konnten im Rahmen dieser Studien Einsparpotenziale von ca. 25-35\% im Rahmen der ärztlichen Behandlung erzielt werden. Der größte Einflussfaktor sind die intensivmedizinischen Kosten (3.011.891€ bzw. 44,5\% der Gesamtkosten). Hier sollten medizinisch sinnvolle Möglichkeiten zur Effizienzsteigerung konsequent umgesetzt werden. Die aktuelle Entwicklung der intensivmedizinischen Versorgung schwer verletzter PatientInnen zeigt beispielsweise einen, medizinisch und wirtschaftlich begrüßenswerten, Rückgang der Beatmungsdauer auf Intensivstationen [27]. Zusätzlich kommt es in den letzten Jahren zunehmend zu einer Reduktion der Gesamtverweildauer im Krankenhaus, was die Kosten für den Verlauf auf der Normalstation ebenfalls reduziert [27].

Sollte sich keine Verbesserung der Kosten-/Erlösstruktur bei der Polytraumaversorgung ergeben, bleibt $\mathrm{zu}$ befürchten, dass durch Selektionieren der angenommenen PatientInnen oder sogar durch Schließungen von Traumazentren versucht werden könnte, erlösoptimiert zu behandeln.

Die Entscheidung zur Versorgung schwer verletzter PatientInnen darf keine finanziell beeinflusste Überlegung sein. Sie ist Teil des Versorgungsauftrags aller lokalen, regionalen und überregionalen Traumazentren. Folglich bleibt es der Politik überlassen, durch eine adäquate Finanzierung von Traumazentren die Überlebensraten und das funktionelle Outcome der Patienten auf dem hohen Niveau zu halten, das wir in Deutsch- land erreicht haben [28, 29] - nicht nur aufgrund ethischer Bedenken, sondern auch weil der volkswirtschaftliche Schaden durch Verlust von Arbeitskraftpotenzial und durch Aufwendungen der gesetzlichen Unfallversicherung wesentlich höher ist.

Die Errechnung der in dieser Studie genannten Kosten basiert auf Schätzungen anhand der den AutorInnen zur Verfügung stehenden Daten (ausführlicher Methodenteil im Zusatzmaterial online: ESM10: Detaillierte Methode, weitere Ergebnisse, weiterführende Literatur). Für einige Positionen mussten hier Annäherungen getroffen werden, die zudem spezifisch für das Universitätsklinikum Leipzig sind und regional unterschiedlich sein können (z. B. Miete). In der Gesamtheit ist der Ansatz zur Kostenschätzung jedoch detaillierter als die der vorangegangenen Studien [12-17].

Für die betriebswirtschaftliche $\mathrm{Be}$ trachtung muss zwischen patientenunabhängigen Kosten (Fixkosten, z.B. Infrastrukturkosten des Schockraums) und patientenabhängigen Kosten (variablen Kosten, z.B. Implantatkosten bei Operationen) unterschieden werden. Um für erbrachte medizinische Behandlungen leistungsgerechte Erlöse mit Fallpauschalen zu vergüten, müsste man die Grenzkosten der Behandlung von schwer verletzten PatientInnen betrachten (Kosten, die durch die Versorgung zusätzlicher Fälle entstehen). Limitierend werden in dieser Studie aber kalkulierte Durchschnittskosten der behandelten PatientInnen untersucht. Diese Limitation relativiert sich, wenn man die ebenfalls retrospektive Datenerhebung des InEK zur Erhebung des Relativgewichts der einzelnen DRG aus den jährlichen Kostendaten der Kalkulationskrankenhäuser in die Überlegung miteinbezieht.

\section{Fazit für die Praxis}

Im angestellten Vergleich von Behandlungskosten für schwer verletzte PatientInnen und der Vergütung nach aG-DRG an einem universitären Maximalversorger und überregionalen Traumazentrum zeigt sich ein erhebliches betriebswirtschaftliches Defizit auf. 
Die Ergebnisse dieser Studie sollen als Diskussionsgrundlage für weitere und unbedingt notwendige Verhandlungen bei der Kalkulation der Fallpauschalen im deutschen DRG-System hin zu einer gerechteren Entlohnung dieser komplexen Behandlungsfälle und Abdeckung der Vorhaltekosten dienen, sollten aber auch zu Überlegungen bezüglich einer kosteneffizienteren Versorgung innerhalb der Traumazentren motivieren.

\section{Korrespondenzadresse

Nikolas Schopow, M.B.A
Klinik für Orthopädie,
Unfallchirurgie und
Plastische Chirurgie,
Universitätsklinikum Leipzig
Liebigstraße 20,04103 Leip-
zig, Deutschland
schopow@medizin.uni-
leipzig.de

Funding. Open Access funding enabled and organized by Projekt DEAL.

\section{Einhaltung ethischer Richtlinien}

Interessenkonflikt. N. Schopow, A. Botzon, K. Schneider, C. Fuchs, C. Josten, N. von Dercks, J. Fakler und G. Osterhoff geben an, dass kein Interessenkonflikt besteht.

Für diesen Beitrag wurden von den Autoren keine Studien an Menschen oder Tieren durchgeführt. Für die aufgeführten Studien gelten die jeweils dort angegebenen ethischen Richtlinien.

Open Access. Dieser Artikel wird unter der Creative Commons Namensnennung 4.0 International Lizenz veröffentlicht, welche die Nutzung, Vervielfältigung, Bearbeitung, Verbreitung und Wiedergabe in jeglichem Medium und Format erlaubt, sofern Sie den/die ursprünglichen Autor(en) und die Quelle ordnungsgemäß nennen, einen Link zur Creative Commons Lizenz beifügen und angeben, ob Änderungen vorgenommen wurden.

Die in diesem Artikel enthaltenen Bilder und sonstiges Drittmaterial unterliegen ebenfalls der genannten Creative Commons Lizenz, sofern sich aus der Abbildungslegende nichts anderes ergibt. Sofern das betreffende Material nicht unter der genannten Creative Commons Lizenz steht und die betreffende Handlung nicht nach gesetzlichen Vorschriften erlaubt ist, ist für die oben aufgeführten Weiterverwendungen des Materials die Einwilligung des jeweiligen Rechteinhabers einzuholen.

Weitere Details zur Lizenz entnehmen Sie bitte der Lizenzinformation auf http://creativecommons.org/ licenses/by/4.0/deed.de.

\section{Literatur}

1. Muhr G, Tscherne H (1978) Bergung und Erstversorgung beim Schwerverletzten. Chirurg 49(10):593-600

2. Pape $\mathrm{H}-\mathrm{C}$, Lefering $\mathrm{R}$, Butcher N, Peitzman A, Leenen L, Marzi I et al (2014) The definition of polytrauma revisited: an international consensus process and proposal of the new 'Berlin definition.' J Trauma Acute Care Surg 77(5):780-786

3. InEK (2019) Abschlussbericht zur Weiterentwicklung des G-DRG-Systems für 2019. https://www.g drg.de/G-DRG-System_2019/Abschlussbericht_ zur_Weiterentwicklung_des_G-DRG-Systems_ und_Report_Browser/Abschlussbericht_zur_ Weiterentwicklung_des_G-DRG-Systems_fuer_ 2019.Zugegriffen: 1. Nov. 2020

4. InEK (2017) Fallpauschalenkatalog 2017

5. InEK (2018) Fallpauschalenkatalog 2018

6. InEK (2019) Fallpauschalenkatalog 2019

7. InEK (2020) Fallpauschalenkatalog 2020. https://www.g-drg.de/G-DRG-System_2020/ Fallpauschalen-Katalog/Fallpauschalen-Katalog 2020.Zugegriffen: 1.Nov. 2020

8. Flohé $S$, Buschmann C, Nabring J, Merguet $P$, Luetkes P, Lefering R et al (2007) Polytraumadefinition im G-DRG-System 2006. Unfallchirurg 110(7):651-658. https://doi.org/10.1007/s00113007-1300-010.1007/s00113-007-1300-0

9. Haas NP, von Fournier C, Tempka A, Südkamp NP (1997) Traumazentrum 2000 Wieviele und welche Traumazentren braucht Europa um das Jahr 2000? Unfallchirurg 100(11):852-858. https://doi.org/ $10.1007 / 5001130050204$

10. Wilke MH, Höcherl E, Scherer J, Janke L (2001) Die Einführung des neuen DRG-basierten Entgeltsystems in Deutschen Krankenhäusern Eine schwierige Operation? Erfahrungen und Lösungsansätze aus der Sicht der Unfallchirurgie. Unfallchirurg 104(5):372-379. https://doi.org/10. 1007/s001130050745

11. Qvick B, Bühren V, Woltmann A (2011) Ist ein Polytrauma heutzutage noch bezahlbar? Unfallchirurg 115:467-468

12. Lefering R, Mahlke L, Franz D (2017) Der Kostenschätzer im TraumaRegister DGU. Unfallchirurg 120(12):1065-1070. https://doi.org/10.1007/ s00113-016-0266-1

13. Grotz M, Schwermann T, Lefering R, Ruchholtz $S$, Graf v.d. Schulenburg JM, Krettek C et al (2004) DRG-Entlohnung beim Polytrauma. Unfallchirurg 107(1):68-75. https://doi.org/10.1007/s00113003-0715-5

14. Mahlke L, Lefering R, Siebert H, WindolfJ, Roeder $\mathrm{N}$, Franz D (2013) Abbildung von Schwerverletzten im DRG-System. Chirurg 84(11):978-986. https:// doi.org/10.1007/s00104-013-2490-3

15. Mahlke L, Schaefer O, Grotz M, Krettek C, Pape H-C (2005) G-DRG-System. Unfallchirurg 107(12):1216-1220. https://doi.org/10.1007/ s00113-004-0897-5

16. Juhra C, Franz D, Roeder N, Vordemvenne T, Raschke MJ (2009) Abbildung des schwer verletzten Patienten im G-DRG-System 2008. Unfallchirurg 112(5):525-532. https://doi.org/10.1007/s00113 009-1570-9

17. Hebler U, Müther M, Muhr G, Gekle C (2007) Ist unfallchirurgische Intensivmedizin noch bezahlbar. Trauma Berufskrankh 9(3):163-166. https://doi. org/10.1007/s10039-007-1248-x

18. Pape H-C, Grotz M, Schwermann T, Ruchholtz S, Lefering R, Rieger M et al (2003) Entwicklung eines Modells zur Berechnung der Kosten der Versorgung schwer Verletzter - eine Initiative des Traumaregisters der DGU. Unfallchirurg 106(4):348-357. https://doi.org/10.1007/s00113003-0605-X

19. Statistisches Bundesamt (2018) Personalkosten deutscher Krankenhäuser im Pflege- und ärztlichem Dienst in den Jahren 2000 bis 2017 (in Millionen Euro). https://de.statista. com/statistik/daten/studie/169305/umfrage/ personalkosten-deutscher-krankenhaeuserpflege-und-aerztlicher-dienst-seit-2000/.Zugegriffen: 1. Nov. 2020

20. Statistisches Bundesamt (2018) Bereinigte Kosten je Behandlungsfall in deutschen Krankenhäusern in den Jahren 1991 bis 2017 (in Euro je Behandlungsfall). https://de.statista.com/statistik/ daten/studie/322429/umfrage/bereinigtekosten-je-behandlungsfall-in-deutschenkrankenhaeusern/.Zugegriffen: 1.Nov. 2020

21. Statistisches Bundesamt (2018) Sachkosten deutscher Krankenhäuser insgesamt in den Jahren 2000 bis 2017 (in Milliarden Euro). https://de.statista.com/statistik/daten/ studie/169301/umfrage/sachkosten-deutscherkrankenhaeuser-insgesamt-seit-2000/. Zugegriffen: 1. Nov. 2020

22. Statistisches Bundesamt (2019) Gesundheitsausgaben in Deutschland nach Einrichtung in den Jahren 2011 bis 2017 (in Millionen Euro). https://de.statista.com/statistik/daten/studie/ 256288/umfrage/gesundheitsausgaben-nacheinrichtung-in-deutschland/. Zugegriffen: 1. Nov. 2020

23. Bouillon B, Pieper D (2016) S3-Leitlinie Polytrauma / Schwerverletzten-Behandlung AWMF Register-Nr. 012/019. https://www. awmf.org/uploads/tx_szleitlinien/012-019|_S3 Polytrauma_Schwerverletzten-Behandlung 2017-08.pdf.Zugegriffen: 1.Nov. 2020

24. DGU (2019) Weißbuch Schwerverletztenversorgung, 3. Aufl. Deutsche Gesellschaft für Unfallchirurgie e. V. (DGU), Berlin

25. Taheri PA, Wahl WL, Butz DA, Iteld LH, Michaels AJ, Griffes LC et al (1998) Trauma service cost: the real stow. Ann Surg 227(5):720-725

26. Taheri PA, Butz D, Griffes LC, Morlock DR, Greenfield LJ (2000) Physician impact on the total cost of care. Ann Surg 231(3):432-435

27. Böhmer $A B$, Poels $M$, Kleinbrahm $K$, Lefering $R$, Paffrath T, Bouillon B et al (2016) Change of initial and ICU treatment over time in trauma patients. An analysis from the TraumaRegister DGU. Langenbecks Arch Surg 401(4):531-540. https://doi.org/10.1007/s00423-016-1428-x

28. Rotondo MF, Bard MR, Sagraves SG, Toschlog EA, Schenarts PJ, Goettler CE et al (2009) What price commitment: what benefit? The cost of a saved life in a developing level I trauma center. J Trauma Acute Care Surg 67(5):915-923

29. Gabbe BJ, Biostat GD, Lecky FE, Bouamra O, Woodford M, Jenks T et al (2011) The effect of an organized trauma system on mortality in major trauma involving serious head injury: a comparison of the United Kingdom and Victoria, Australia. Ann Surg 253(1):138-143 